\title{
On the Design of Optimal Feedback Control for Systems of Second Order
}

\author{
Alexander M. Formalskii \\ Institute of Mechanics of Moscow Lomonosov State University, Michurinskii prospect, Moscow, Russia \\ E-mail:formal@imec.msu.ru \\ Received May 9, 2010; revised August 14, 2010; accepted August 17, 2010
}

\begin{abstract}
A difficult but important problem in optimal control theory is the design of an optimal feedback control, i.e., the design of an optimal control as function of the phase (state) coordinates [1,2]. This problem can be solved not often. We study here the autonomous nonlinear system of second order in general form. The constraints imposed on the control input can depend on the phase (state) coordinates of the system. The goal of the control is to maximize or minimize one phase coordinate of the considered system while other takes a prescribed in advance value. In the literature, optimal control problems for the systems of second order are most frequently associated with driving both phase coordinates to a prescribed in advance state. In this statement of the problem, the optimal control feedback can be designed only for special kind of systems. In our statement of the problem, an optimal control can be designed as function of the state coordinates for more general kind of the systems. The problem of maximization or minimization of the swing amplitude is considered explicitly as an example. Simulation results are presented.
\end{abstract}

Keywords: System of Second Order, Optimal Feedback Control, Design, Swing, Rocking, Damping, Simulation

\section{Mathematical Model of the Considered System}

Let the motion of the studied object under control be governed by a system of two nonlinear autonomous differential equations of the form

$$
\dot{x}=f_{1}(x, y, u), \quad \dot{y}=f_{2}(x, y, u),
$$

the dot denotes, as usual, derivative with respect to time.

For example, a controllable mechanical system with one degree of freedom is described by similar differential equations. In this case, $x$ is positional coordinate and

$$
f_{1}(x, y, u)=y
$$

is the velocity of the object (linear or angular), function $f_{2}(x, y, u)$ is the generalized force divided by the object's mass or moment of inertia.

Let for each piecewise continuous vector function $u(t)$, system (1) with initial conditions from some region of the phase plane $(x, y)$ has a unique solution $x(t)$, $y(t)$. We assume that the control parameter $u$ belongs to a given set $U(x, y)$ depending on the state coordinates $x$ and $y$. In other words, a vector piecewise con- tinuous function $u(t)$ is assumed to be an admissible control, if

$$
u(t) \in U(x(t), y(t)) .
$$

Here $x(t), y(t)$ is the solution of Equations (1) with $u=u(t)$. If set $U(x, y)$ depends on state coordinates $x, y$, then condition (3) can be checked for a given piecewise continuous control function $u(t)$, in general, only by finding the solution of the system (1) with this control.

Assume in what follows that function $f_{1}(x, y, u)$ does not vanish. To be definite, let

$$
f_{1}(x, y, u)>0 .
$$

Under condition (4), the coordinate $x$ can only increase with time. If (1) is a mathematical model of a mechanical system with one degree of freedom, then equality (2) takes place and inequality (4) holds in upper half of the phase plane $(x, y)$.

We rewrite system (1) in the form of a first-order equation

$$
\frac{d y}{d x}=\frac{f_{2}(x, y, u)}{f_{1}(x, y, u)}=f(x, y, u) .
$$


Let

$$
x(0)=x_{0}, y(0)=y_{0}
$$

be initial conditions for system (1) or Equation (5). To be definite, assume that $y_{0}>0$.

We do not formulate here in the first Section all conditions on the system (1), on the set $U(x, y)$ (see relation (3)). It is difficult to specify in advance all these conditions. We formulate new assumptions during the problem consideration as need arises.

\section{Sets of Reachability}

Assume that, in the phase plane $(x, y)$, every trajectory $y(x)$ that starts from point (6) and corresponds to an admissible control function $u(t)$, intersects the axis $Y=0$ at some finite time $t$ and for some finite coordinate $x$. Note that time $t$ and coordinate $x$ have their own values for each admissible control function $u(t)$. Consider the set of all possible admissible control functions $u(t)$ and the set of corresponding trajectories $y(x)$, obtained under these controls. More precisely, consider only the portions of these trajectories that start from point (6) and terminate on the axis of abscissas $Y=0$. The collection of these curves covers a set of points that form a reachable set [3] or so-called integral funnel [4,5]. This set of reachability $D$ is schematically shown in Figure 1.

\section{Boundaries of Reachable Set}

Let us consider control that maximizes derivative $\frac{d y}{d x}$ over variable $u$ at the point $(x, y)$. This control maximizes the function $f(x, y, u)$ over argument $u$ on the right-hand side of Equation (5) and it has the form

$$
u=u_{\max }(x, y)=\arg \left[\max _{u \in U(x, y)} f(x, y, u)\right] \text {. }
$$

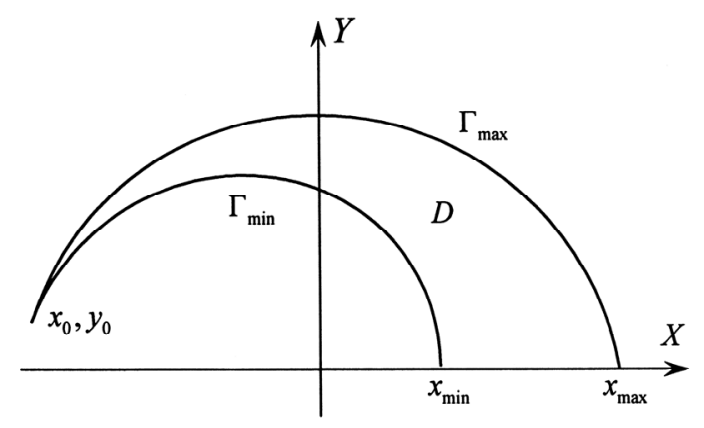

Figure 1. Reachabe set $D$.
We assume here function $f(x, y, u)$ and set $U(x, y)$ such that the maximum in (7) exists and is unique in each point of the phase plane in some domain including the reachable set $D$. We assume also that the solution to system (1) with initial conditions (6) under control (7) yields a piecewise continuous function $u(t)$, i.e., an admissible control function. Let $y=y_{\max }(x)$ be the solution to the equation

$$
\frac{d y}{d x}=f\left[x, y, u_{\max }(x, y)\right]
$$

with initial conditions (6). Denote by $\Gamma_{\max }$ the part of the trajectory $y=y_{\max }(x)$ for $x_{0} \leq x \leq x_{\max }$, where $x_{\max }$ is the first value of argument $x$, at which function $y=y_{\max }(x)$ vanishes $\left(y_{\max }(x)=0\right)$. Now we will show that the curve $\Gamma_{\max }$ is the upper boundary of reachable set $D$ (see Figure 1).

Given any control function $u^{*}(x, y) \neq u_{\max }(x, y)$, assume that the trajectory of Equation (5) starting from some point $(x, y) \in \Gamma_{\max }$, lies above by curve $\Gamma_{\max }$. Then, at this point, we have the inequality

$$
f\left[x, y, u^{*}(x, y)\right] \geq f\left[x, y, u_{\max }(x, y)\right],
$$

or the solution $y=y_{\max }(x)$ of Equation (8) is not unique. However, inequality (9) contradicts condition (7), while the solution $y=y_{\max }(x)$ of Equation (8) starting at point (6) is unique by assumption.

Now consider control function that minimizes derivative $\frac{d y}{d x}$ over parameter $u$ at the point $(x, y)$, i.e., minimizes the function $f(x, y, u)$ over argument $u$ on the right-hand side of Equation (5):

$$
u=u_{\min }(x, y)=\arg \left[\min _{u \in U(x, y)} f(x, y, u)\right] \text {. }
$$

We assume here function $f(x, y, u)$ and set $U(x, y)$ such that the minimum in (10) exists and is unique in the phase plane in some domain including the reachable set $D$. Let the solution to system (1) with initial conditions (6) under control (10) yields a piecewise continuous function $u(t)$, i.e., an admissible control function.

Let $y=y_{\min }(x)$ be the solution to the equation

$$
\frac{d y}{d x}=f\left[x, y, u_{\min }(x, y)\right]
$$

with initial conditions (6). Denote by $\Gamma_{\min }$ the part of the trajectory $y=y_{\min }(x)$ for $x_{0} \leq x \leq x_{\min }$, where $x_{\min }$ is the first value of argument $x$, at which function $y=y_{\min }(x)$ vanishes $\left(y_{\min }(x)=0\right)$.

Applying the considerations similar to that used for the curve $\Gamma_{\max }$, we can prove that curve $\Gamma_{\min }$ is the lower boundary of reachable set $D$. 


\section{Statement of the Problem and its Solution}

The problem is to find an admissible control, under which the coordinate $x$ reaches its maximum when the coordinate $y$ vanishes at first time (from the beginning of the motion). This maximization problem can be symbolically written as

$$
\underset{\boldsymbol{u} \in U(x, y)}{\max }[x] \text { at } y=0 .
$$

It should be accented that coordinate $x$ has to be maximized not at a prescribed time but at the time when coordinate $y$ vanishes. When Equations (1) describe the motion of a mechanical system with one degree of freedom and equality (2) holds, the condition $y=0$ means that the velocity of motion vanishes. In this case, the goal is to maximize the deviation of the $x$-coordinate from the initial position by the time when the velocity of motion vanishes.

Along with the formulated above problem, we also consider problem to find an admissible control, under which the coordinate $x$ reaches its minimum when the coordinate $y$ vanishes at first time (from the beginning of the motion):

$$
\min _{\mathbf{u} \in U(x, y)}[x] \text { at } y=0 .
$$

A maximization problem of type (12) was considered, for example, in paper [6] and in other works of the same authors. In these works, the conditions for the absolute stability of bilinear systems were found, by constructing a control that maximally "swings" the system.

The analysis performed in Section 3 implies that in the reachable set $D$, there is no a trajectory with so large $x$-coordinate as $x_{\max }$ (see Figure 1). Consequently the maximum value of $x$-coordinate is equal to $x_{\max }$ and the control $u=u_{\max }(x, y)$ (see formula (7)) solves problem (12).

The control $u=u_{\min }(x, y)$ (see formula (10)) solves problem (13), and the minimal value of $x$-coordinate is equal to $x_{\min }$ (see Figure 1).

\section{More General Case}

More general problems than those discussed above are the maximization or minimization of the coordinate $x$ at the time when coordinate $y$ first takes a prescribed in advance value $\bar{y}$, which may be nonzero. If $\bar{y}<y_{0}$, then, as before, the control $u=u_{\max }(x, y)$ (see formula (7)) is optimal for the maximization problem, while the control $u=u_{\min }(x, y)$ (see formula (10)) is optimal for the minimization problem. If $\bar{y}>y_{0}$ (see Figure 2), then the maximum of the coordinate $x$ is reached under

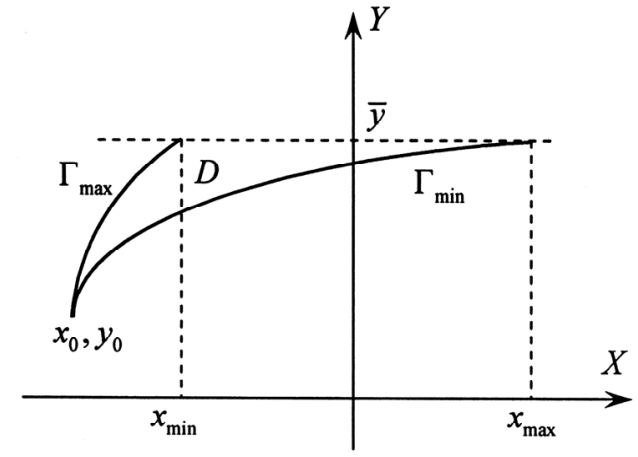

Figure 2. Reachabe set $D$.

the control $u=u_{\min }(x, y)$, while the minimum of the coordinate $x$ is reached under the control $u=u_{\max }(x, y)$. This is explained by the fact that, for $\bar{y}>y_{0}$, the upper boundary $\Gamma_{\max }$ of the reachable set $D$ intersects the line $y=\bar{y}$ at a smaller value of coordinate $x$, than the lower boundary $\Gamma_{\min }$.

Formulas (7) or (10) can be considered for the formulated above problems as a local maximum or minimum principle.

In the next section, the obtained above results are illustrated by constructing an optimal control of the motion of the swing.

\section{Maximization and Minimization of the Swing Amplitude}

As a model of a swing with a human on it, we consider a physical pendulum of mass $\mu$ with a point particle of mass $M$ moving along it (see Figure 3).

In Figure 3, $x$ is the deflection angle of the pendulum from the vertical line (we consider that $-\pi<x<\pi$ ), $u$ denote the distance $O M$ between points $M$ and $O$ $(u=O M)$. Assume that distance $u$ is a control parameter. Unlike the general case, here this control parameter $u$ is a scalar. The distance $u$ can vary within bounded limits:

$$
u_{0} \leq u \leq u_{1}\left(u_{0}, u_{1}=\text { const }, u_{0}<u_{1}\right) .
$$

Let $J$ denote the moment of inertia of the pendulum (without point participle $M$ ) relative to the point of suspension $O, \rho$ denote the distance from the suspension joint $O$ to the pendulum's center of mass $C(\rho=O C), g$ is the gravity acceleration.

According to the principle of moment of angular momentum relative to joint $O$, the nonlinear equation of motion of the swing has the form [7]:

$$
\frac{d}{d t}\left[\left(J+M u^{2}\right) \frac{d x}{d t}\right]=-(M u+\mu \rho) g \sin x-c \frac{d x}{d t} .
$$

Here $\left(J+M u^{2}\right) \dot{x}$ is the angular momentum of the 


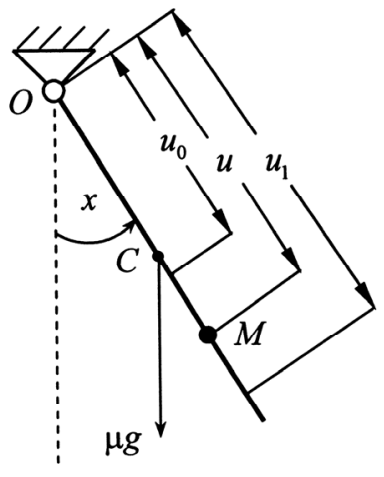

Figure 3. The scheme of the swing.

system relative to suspension point $O, c$ is the coefficient of viscous resistance (for example, viscous friction in the suspension joint $O$ ).

Let $y$ denote the angular momentum $\left(J+M u^{2}\right) \dot{x}$. Then the second-order Equation (15) can be rewritten as a system of two first-order equations of form (1):

$$
\begin{aligned}
& \dot{x}=\frac{y}{J+M u^{2}}, \\
& \dot{y}=-(M u+\mu \rho) g \sin x-\frac{c y}{J+M u^{2}} .
\end{aligned}
$$

Let the initial state of system (16) be specified as

$$
x(0)<0, y(0)=0 \text {. }
$$

The problem is to find a law of variation of the distance $u$ subject to inequalities (14) at which the deflection of the angle $x$ is maximal at the instant $\theta$ when angular momentum $y$ (and, hence, the velocity $\dot{x}$ ) vanishes $(y(\theta)=0, \dot{x}(\theta)=0)$ first from the start of the motion. In other words, the goal is to maximize the deviation of the swing from the vertical line (amplitude of the swing) at the end of the first half-period of its oscillations. We consider initial value $x(0)$ of angle $x$ sufficiently close to zero and assume that for this angle $x(0)$ and each admissible control $u(t)$ the corresponding instant $\theta$ exists when $y(\theta)=0$.

It follows from second Equation (16) that $y>0$ (i.e., $\dot{x}>0$ ), if $0<t<\theta$. Then on the time interval $0<t<\theta$, system (16) can be rewritten in a form similar to (5), namely

$$
\frac{d y}{d x}=-c-\frac{(M u+\mu \rho)\left(J+M u^{2}\right) g \sin x}{y} .
$$

According to the above results, maximizing the right-hand side of Equation (18) over argument $u$ on interval (14) yields an optimal control law for the swing on a half-period of oscillations for which $y>0$

$$
u= \begin{cases}u_{1}, & \text { if } x<0 \\ u_{0}, & \text { if } x>0\end{cases}
$$

This control law means that distance $u=O M$ is maximal as possible when $x<0$ and minimal as possible when $x>0$.

The next (second) half-period of oscillations, for which $y<0$ (i.e., $\dot{x}<0$ ), can be considered by analogy with the first half-period. As a result, we conclude that the optimal control of the swing on each half-period is described by the relations

$$
u= \begin{cases}u_{1}, & \text { if } x \dot{x}<0 \\ u_{0}, & \text { if } x \dot{x}>0 .\end{cases}
$$

Figure 4 shows in the phase plane $(x, \dot{x})$ the synthesis picture of optimal rocking control $u(x, \dot{x})$ (19). Under control (19), the point particle $M$ instantaneously moves up to the stop when the swing goes through the lower position and moves down to the stop when the swing maximally deviates from the vertical, i.e., when its angular velocity $\dot{x}$ vanishes. Thus, the problem of optimal rocking of the swing is solved.

The control given by formula (19) was considered in the book [8] but without any discussion of its optimality.

Now let us consider the problem of optimal damping of the swing at the end of each half-period of its oscillations. To design the optimal damping control we have to solve the problem (13) for the right-hand side of Equation (18). After solving this problem we conclude that the deviation of the swing from the vertical at the end of each oscillation half-period is minimal under the control:

$$
u= \begin{cases}u_{1}, & \text { if } x \dot{x}>0 \\ u_{0}, & \text { if } x \dot{x}<0 .\end{cases}
$$

In Figure 5, the synthesis picture of optimal damping

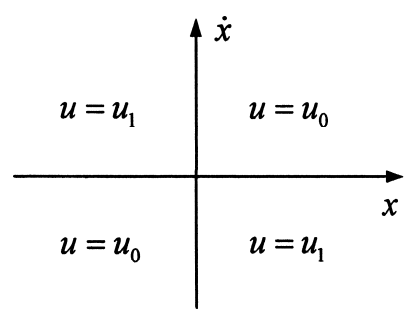

Figure 4. Design of the optimal rocking feedback control.

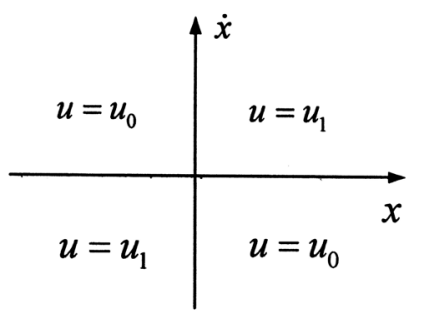

Figure 5. Design of the optimal damping feedback control. 
control $u(x, \dot{x})(20)$ is shown in the phase plane $(x, \dot{x})$. Under control (20), the point particle $M$ instantaneously moves down to the stop when the swing goes through the lower position and moves up to the stop when the swing maximally deviates from the vertical, i.e., when its angular velocity $\dot{x}$ vanishes.

So, the feedback control law (20) for the optimal damping of the swing is contrary to the feedback control law (19) for the optimal rocking of the swing.

\section{Simulation of the Optimal Swing Motion}

Consider Equations (16) with the following numerical parameters:

$$
\begin{aligned}
& \mu=5 \mathrm{~kg}, J=26.67 \mathrm{~kg} \cdot \mathrm{m}^{2}, M=70 \mathrm{~kg}, u_{0}=3 \mathrm{~m}, \\
& u_{1}=3.75 \mathrm{~m}, \rho=2 \mathrm{~m}, \mathrm{c}=2 \mathrm{~N} \cdot \mathrm{m} \cdot \mathrm{s} .
\end{aligned}
$$

Here we assume that pendulum is a homogeneous beam of the length $2 \rho=4 m$, and consequently

$$
J=\frac{1}{3} \mu(2 \rho)^{2}=\frac{4}{3} \mu \rho^{2} .
$$

In Figure 6, the graphs of angle $x$, of angular velocity $\dot{x}$, of control $u$ as functions of time are shown. These functions are obtained solving equations of motion (16) under optimal rocking feedback control (19) with parameters $(21)$ and initial conditions $x(0)=-0.1, y(0)=0$.

Figure 6 shows that under control $u(x, \dot{x})$ (19) the amplitude of the swing increases. The relay control function $u(t)$ instantaneously switches from value $u_{1}$ to value $u_{0}$ when angle $x$ becomes zero and switches from value $u_{0}$ back to value $u_{1}$ when the velocity $\dot{x}$ becomes zero. Between the shift points, control parameter $u=$ const .

Figure 7 shows the phase portrait of the rocking motion in the plane $(x, \dot{x})$.

In Figures 6 and 7, we observe the jumps of angular velocity $\dot{x}$ at the instants when control $u(t)$ switches from value $u_{1}$ to value $u_{0}$. At these instants, the moment of inertia $J+M u^{2}$ of the swing together with the point particle $M$ (with a human) decreases and the angular velocity $\dot{x}$ increases due to the conservation of the angular momentum $y=\left(J+M u^{2}\right) \dot{x}$, which is not zero at these times.

In Figure 8, the graphs of angle $x$, of angular velocity $\dot{x}$, of control $u$ as functions of time are shown. These functions are obtained solving equations of motion (16) under optimal damping feedback control (20) with parameters $(21)$ and initial conditions $x(0)=-\pi / 2 \approx-1.57$, $y(0)=0$.

Figure 8 shows that under control $u(x, \dot{x})$ (19) the amplitude of the swing decreases. The relay control function $u(t)$ instantaneously switches from value $u_{1}$ to
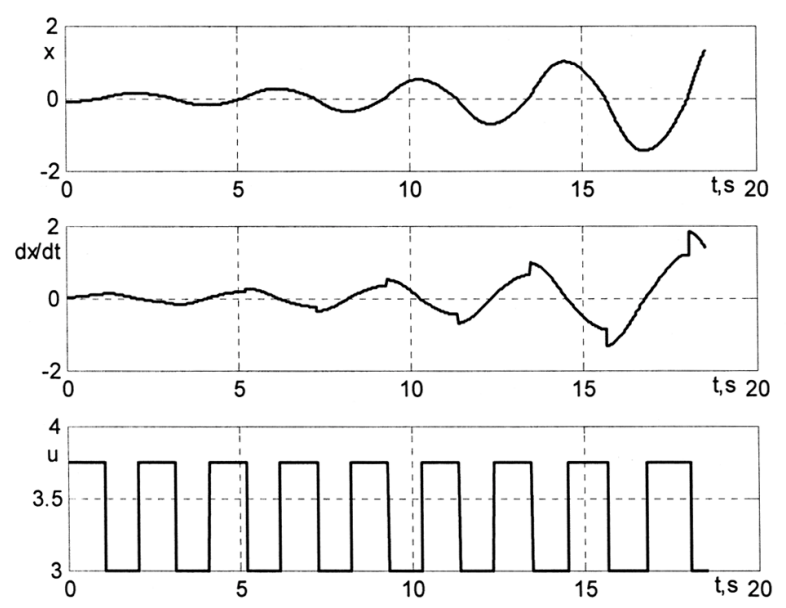

Figure 6. Graphs of angle $x(t)$, angular velocity $\dot{x}(t)$ and function $u(t)$ for rocking feedback control.

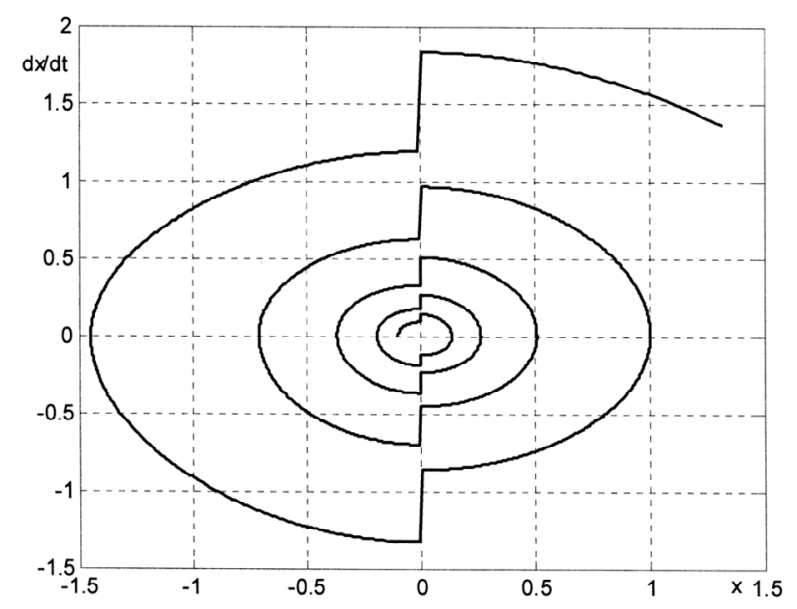

Figure 7. Phase portrait in the plane $(x, \dot{x})$ for rocking feedback control.
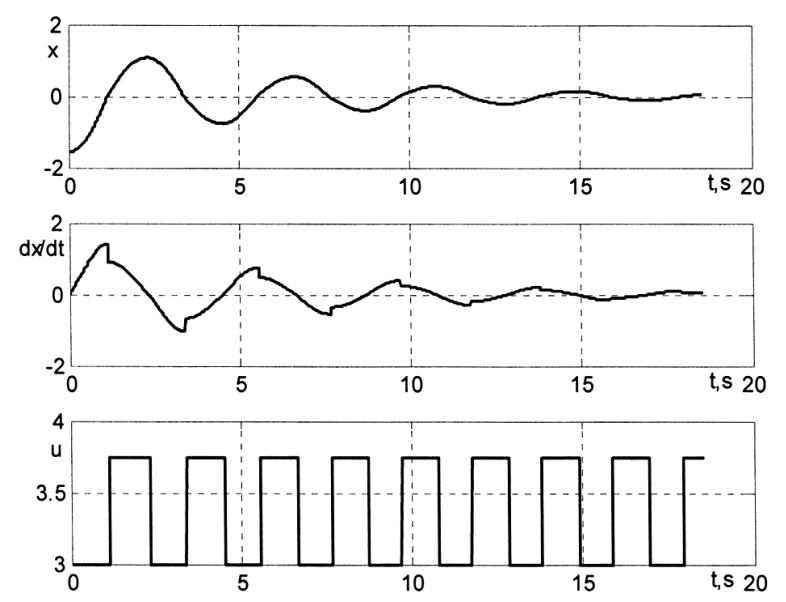

Figure 8. Graphs of angle $x(t)$, angular velocity $\dot{x}(t)$ and function $u(t)$ for damping feedback control. 


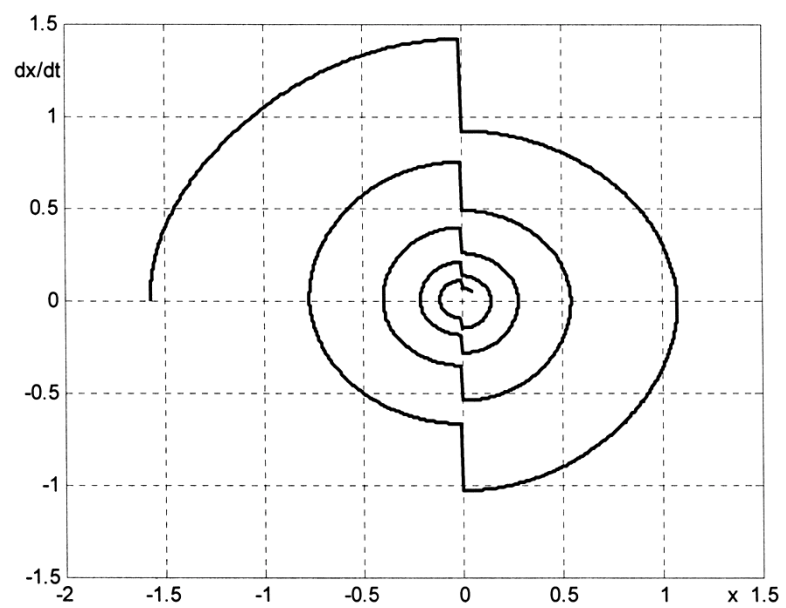

Figure 9. Phase portrait in the plane $(x, \dot{x})$ for damping feedback control.

value $u_{0}$ when the velocity $\dot{x}$ becomes zero, and switches in the opposite direction when angle $x$ becomes zero. Between the shift points, the distance $u$ remains constant.

Figure 9 shows the phase portrait of the damping motion in the plane $(x, \dot{x})$.

In Figures 8 and 9, we observe the jumps of angular velocity $\dot{x}$ at the times when control $u(t)$ switches from value $u_{0}$ to value $u_{1}$. At these times, the moment of inertia $J+M u^{2}$ of the swing together with the point particle $M$ (with a human) increases instantaneously and the angular velocity $\dot{x}$ decreases also instantaneously due to the conservation of the angular momentum $y=\left(J+M u^{2}\right) \dot{x}$, which is not zero.

\section{Acknowledgements}

This work has been carried out with financial support from the Russian Foundation for Basic Research, Grants 07-01-92167, 09-01-00593-a, 10-07-00619-a.

\section{References}

[1] L. S. Pontryagin, V. G. Boltyanskii, R. V. Gamkrelidze, and E. F. Mischenko, "The Mathematical Theory of Optimal Processes," Wiley Interscience, New York, 1962.

[2] V. G. Boltyanskii, "Mathematical Methods of Optimal Control," Holt, Rinehart \& Winston, 1971.

[3] A. M. Formalskii, "Controllability and Stability of Systems with Restricted Control Resources," Nauka, Moscow, 1974 (in Russian).

[4] I. A. Sultanov, "Studying the Control Processes Obeying Equations with Underdefinite Parameters," Automation and Remote Control, No. 10, October 1980, pp. 30-41.

[5] A. G. Butkovskiy, "Phase Portrait of Control Dynamical Systems," Kluwer, 1991

[6] V. V. Alexandrov and V. N. Jermolenko, "On the Absolute Stability of Second-Order Systems," Bulletin of Moscow University, Series 1, Mathematics and Mechanics, No. 5, October 1972, pp. 102-108.

[7] E. K. Lavrovskii and A. M. Formalskii, "Optimal Control of the Pumping and Damping of a Swing," Journal of Applied Mathematics and Mechanics, Vol. 57, No. 2, April 1993, pp. 311-320.

[8] K. Magnus, "Schwingungen Eine einfurung in die theoretische behandlung von schwingungsproblemen,” D. G. Teubner Stuttgart, 1976. 